\title{
Nanostructured Porous Silicon - Optical Properties, Surface Modification and Sensor Applications
}

\author{
Ivan Jelínek ${ }^{a \star}$, Tomáš Chvojka ${ }^{a}$, Vladimír Vrkoslava ${ }^{a}$, Jindřich Jindřich ${ }^{b}$, Miroslav Lorenc ${ }^{b}$, \\ Daniel Nižňanskýc, Ivan Němec ${ }^{c}$, Vladimír Králd, and Juraj Diane
}

\begin{abstract}
Porous silicon is a very attractive material due to its intense visible photoluminescence at room temperature. Its unique physical and chemical properties are determined by the porous structure where a nanosized silicon layer is formed during the electrochemical etching of crystalline silicon. So far, the high chemical reactivity of the porous silicon surface has prevented its extensive application in optoelectronics. Considerable effort has been put into the development of suitable techniques of stabilization. On the other hand, high chemical reactivity and sorption capacity of porous silicon represent the principal advantage for the construction of sensitive sensors of chemical species. This article provides a brief overview of the ongoing activities in the development of poroussilicon-based chemical sensors at the Charles University and at the Institute of Chemical Technology. So far, the detection of chemical species is based on the measurement of the changes in photoluminescence intensity and photoluminescence decay time, other complementary electrochemical and optical detection methods will follow in order to increase the selectivity of the detection. Another approach resulting in increased selectivity of sensor response utilizes the modification of porous silicon surface - either by physical adsorption or chemical derivatization - by various molecules with recognition properties. Due to the interdisciplinary nature of the research the involved scientists have built a network of collaborating laboratories specialized in various fields - material research, surface chemistry, design and synthesis of the molecules with recognition properties and sensor development.
\end{abstract}

Keywords: Optical sensor · Photoluminescence quenching $\cdot$ Porous silicon $\cdot$ Recognition enhancement

${ }^{*}$ Correspondence: Dr. I. Jelínek

aDepartment of Analytical Chemistry Faculty of Sciences

Charles University Prague

Hlavova $8 / 2030$

Prague 2, 12842

Czech Republic

E-Mail: ijelinek@natur.cuni.cz

${ }^{b}$ Department of Organic Chemistry

Charles University Prague

Czech Republic

'Department of Inorganic Chemistry

Charles University Prague

Czech Republic

dDepartment of Analytical Chemistry

Institute of Chemical Technology

Prague

Czech Republic

eDepartment of Chemical Physics and Optics

Faculty of Mathematics and Physics

Charles University Prague

Czech Republic

\section{Introduction}

Porous silicon (PS) has attracted much attention since the discovery of its efficient visible room temperature photoluminescence (PL) in 1990 [1]. The principal mechanism responsible for visible PL was attributed to quantum confinement in silicon nanostructures. The enormous interest in this material in the first years of the 21 st century was promoted by the search for efficient visible luminescence silicon-based material for optoelectronic applications. When the decisive role of the PS surface [2] and chemical composition of the surrounding environment [3] on the physical properties of PS was revealed, the attention shifted towards PS surface chemistry [4].

Physical and chemical properties of porous silicon are determined by the morphology of silicon nanostructures (pore size, orientation, and size distribution). Correlation of observed physical properties with morphology of PS films [5] and the relationship between PS morphology and anodization conditions of p-type silicon have been extensively studied. Principal factors influencing the formation rate of the PS layer and its morphology were found. PS macropore formation depends both on the properties of the silicon substrate (crystal orientation [6], doping density [7]) and the experimental conditions for anodization (composition of etching solution, current density [8][9], temperature [10]).

Nowadays, a growing area of PS research is aimed at the development of chemical and biochemical sensors [4]. Besides favorable sensor properties the principal advantage of PS is its material compatibility with common silicon-based microelectronics. Improvement of selectivity and operation stability of PS sensor is an essential prerequisite determining its practical utilization [11]. It is usually accomplished by the attachment of suitable species to the PS surface using various chemical routes [12][13]. Species with recognition properties are often of very high molecular weight or even supramolecular structures (enzymes, antibodies, membranes, or whole cells [14]). In order to achieve sufficiently high coverage of PS surface through chemical bonds, information about pore size, orientation and 
distribution is crucial. Knowledge of the dependence of PS morphology on preparation conditions thus plays a decisive role in the search for effective derivatization methods of PS surfaces for sensor applications.

\section{Experimental}

PS samples were prepared by anodic etching of crystalline $\mathrm{Si}$ wafer $(\mathrm{CZ}$, boron doped, (100), $10 \Omega \mathrm{cm}$, ON Semiconductors) in a HF (50 wt.\%) and absolute ethanol mixture, composition varied from 3:1 to $1: 3$ volume ratio. The current density varied within the interval $1-20 \mathrm{~mA} \mathrm{~cm}$, anodization time was $60 \mathrm{~min}$. Porosity and thickness of PS samples were determined by gravimetric methods [15]. Infrared spectra were measured by means of a Fourier transform Nicolet Magna 760 spectrometer operated in transmission mode. The morphology of PS samples was observed by means of a JEOL JSM 6700F NT scanning electron microscope (SEM); the detailed structure was determined by means of a 200 kV TOPCON high resolution transmission electron microscope (HR TEM).

Gas-phase measurements of the photoluminescence PS sensor response were performed in a laboratory-made bulk optode operating with nitrogen as a carrier gas. Photoluminescence was excited either by UV LED (RLT370-10, $370 \mathrm{~nm}, \sim 1 \mathrm{~mW} /$ $\mathrm{cm}^{2}$ ) or a cw Ar${ }^{+}$-laser (457.9 nm, $30 \mu \mathrm{W}$ $\mathrm{cm}^{-2}$ ), collected in a perpendicular direction by means of a glass fiber connected to a emission monochromator, detected by a photomultiplier and processed with a Stanford SR830 lock-in amplifier. Measurements of the photoluminescence sensor response of porous silicon in a liquid phase were performed in a slightly modified setup. The porous silicon sample was fixed in a bulk optode immersed in n-hexane. The excitation beam and photoluminescence were directed to and from the porous silicon sample by means of a fiber optics luminescence probe.

\section{Results and Discussion}

\subsection{Dependence of Porous Silicon Morphology and Chemical Compo- sition on Fabrication Parameters}

Photoluminescence properties of PS are closely related to the sample morphology and porosity. We performed a detailed study of the dependence of these parameters on fabrication conditions. PS samples were prepared at various HF concentrations and current densities and the resulting porosity, thickness, morphology and photoluminescence were studied [16]. Based on experimental results, we found two effective ways of controlling the sample porosity - through the HF concentration and through the current density. However, no direct link between photoluminescence properties (spectral distribution, position of spectral maximum) and total porosity was observed.

According to the quantum confinement mechanism, with increasing porosity of PS the mean size of silicon nanostructures is expected to become smaller and the PL spectral maximum should shift to the blue. Photoluminescence spectra of selected PS samples are presented in Fig. 1. We can see that the PL maximum shifts substantially to lower wavelengths with decreasing HF concentration in the etching solution (Fig 1a); the observed PL blue shift due to the increased current density is about one order of magnitude smaller (Fig. 1b). On the basis of the experimental data obtained we can conclude that the total porosity of the porous layer is not directly linked with the mean size of silicon nanostructures that are responsible for visible photoluminescence.

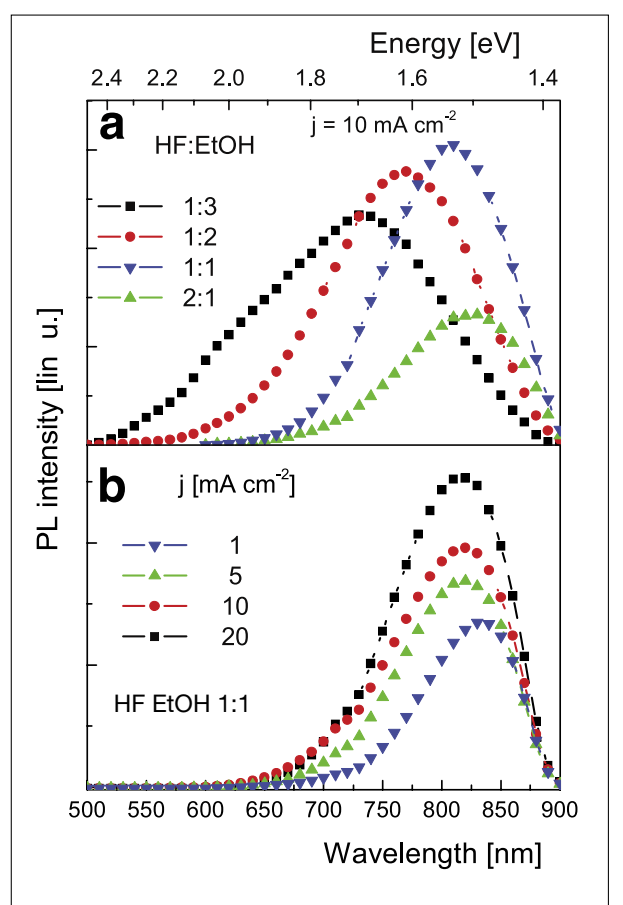

Fig. 1. (a) Room temperature photoluminescence spectra of porous silicon films prepared at various $\mathrm{HF}$ concentrations in etching solutions (current density $10 \mathrm{~mA} \mathrm{~cm}{ }^{-2}$, anodization time $60 \mathrm{~min}$ ); (b) photoluminescence spectra of porous silicon films prepared at various current densities (HF/ ethanol $=1: 1$, anodization time $60 \mathrm{~min}$ ). Spectra were excited with a $\mathrm{Ar}^{+}$laser $(457.9 \mathrm{~nm}$, excitation power $\sim 30 \mu \mathrm{W} \mathrm{cm}^{-2}$ ).

Observed PL maximum shifts for PS prepared under different conditions were also correlated with PS surface morphology as observed by electron microscopy. We may conclude that PS samples prepared at the same HF concentration revealed simi- lar morphology under the electron microscope (not shown) irrespective of the selected current density, whereas substantial differences in morphology of the porous layer were observed for samples prepared at different HF concentrations. Fig. 2 shows cross-sectional SEM views of three samples prepared at $\mathrm{HF}$ concentrations $12.5 \%, 16.7 \%$, and $25 \%$ (current density $10 \mathrm{~mA} \mathrm{~cm}{ }^{-2}$, anodization time $60 \mathrm{~min}$ ) - samples are denoted A, B, and C. For PS sample A the pores were wide with the walls partially cracked. For sample B prepared at higher HF concentration the pores were substantially thinner, which was reflected in a relatively large red shift in the PL spectrum (see Fig. 1). The finest, but most easily destructible porous structure was observed for PS sample C prepared in the most concentrated HF solution. Accordingly, this sample shows the most significant red shift of the PL maximum. A similar relation between sample porosity and PL shift was reported by Bessaïs et al. [17].
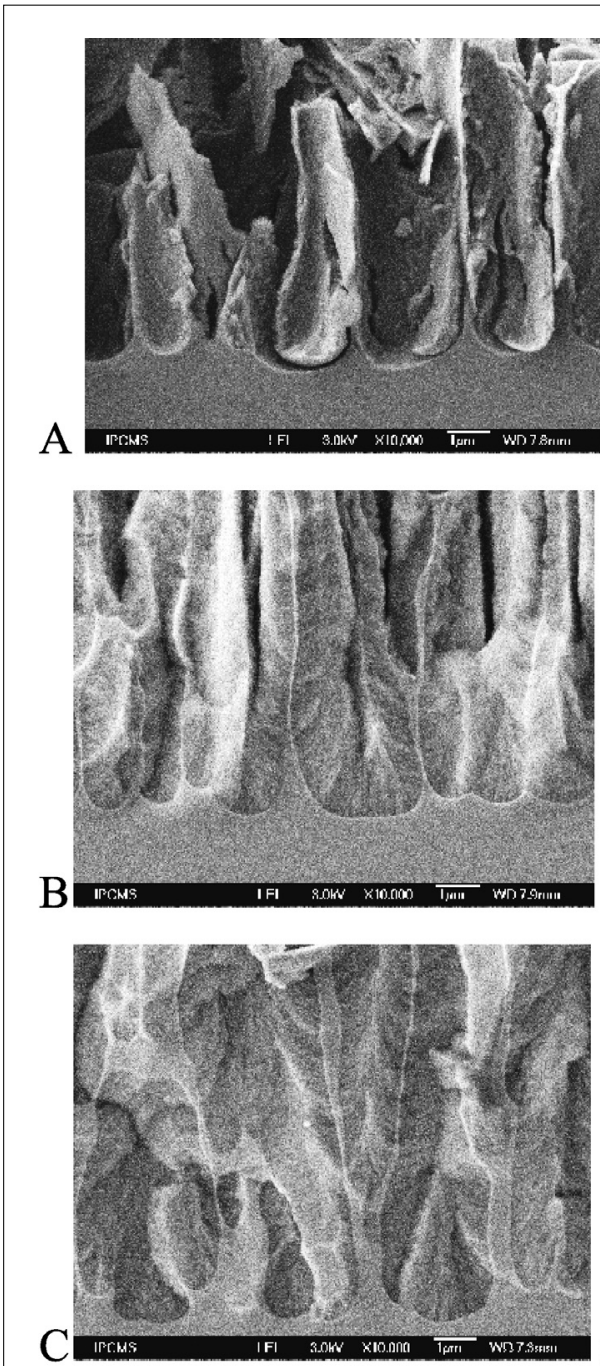

Fig. 2. Cross-sectional electron micrographs of porous films and details of pore morphology for porous silicon samples $A, B$, and $C$ 


\subsection{Sensor Response of Porous \\ Silicon in Gas- and Liquid Phases}

The photoluminescence sensor response of PS was measured for a homological set of linear alcohols from methanol to 1-hexanol in gas and liquid phases. The measurement was accomplished by recording the photoluminescence intensity at selected wavelengths as a function of elapsed time when various amounts of analytes were repeatedly introduced and rinsed out of the system. As examples, time records of the photoluminescence intensity for 1-hexanol in gas phase and methanol in liquid phase are shown in Fig. 3 and Fig. 4.

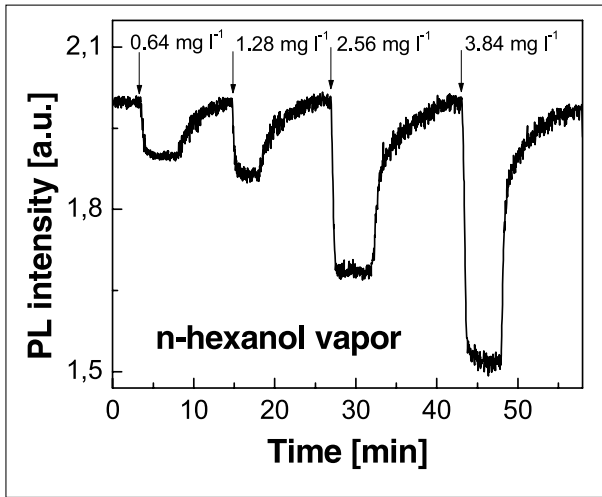

Fig. 3. Variations of photoluminescence intensity of standard porous silicon in the presence of various amounts of 1-hexanol in the gas phase. Emission wavelength $\lambda=700 \mathrm{~nm}$.

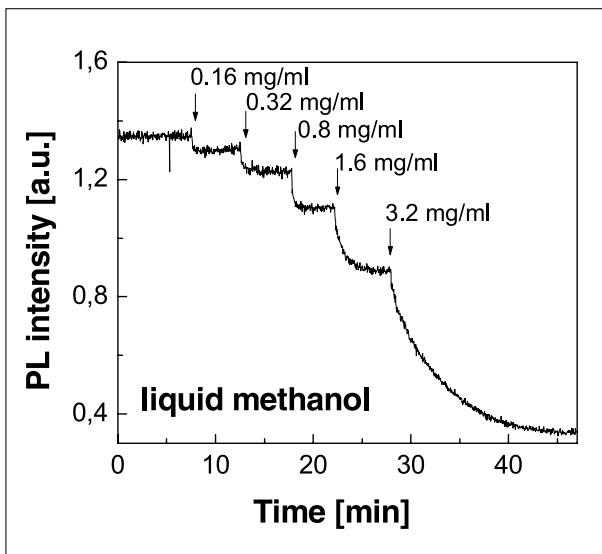

Fig. 4. Variations of photoluminescence intensity of standard porous silicon in the presence of various amounts of methanol in the liquid phase. Emission wavelength $\lambda=700 \mathrm{~nm}$.

The magnitude of photoluminescence quenching depends on analyte concentration. From the time records of photoluminescence intensity we determined calibration curves - a relative decrease of PL intensity as a function of analyte concentration (see Fig. 5 for analytes in gas phase and Fig. 6. for analytes in liquid phase). From the linear part of the calibration curves we determined the fundamental sensor param-
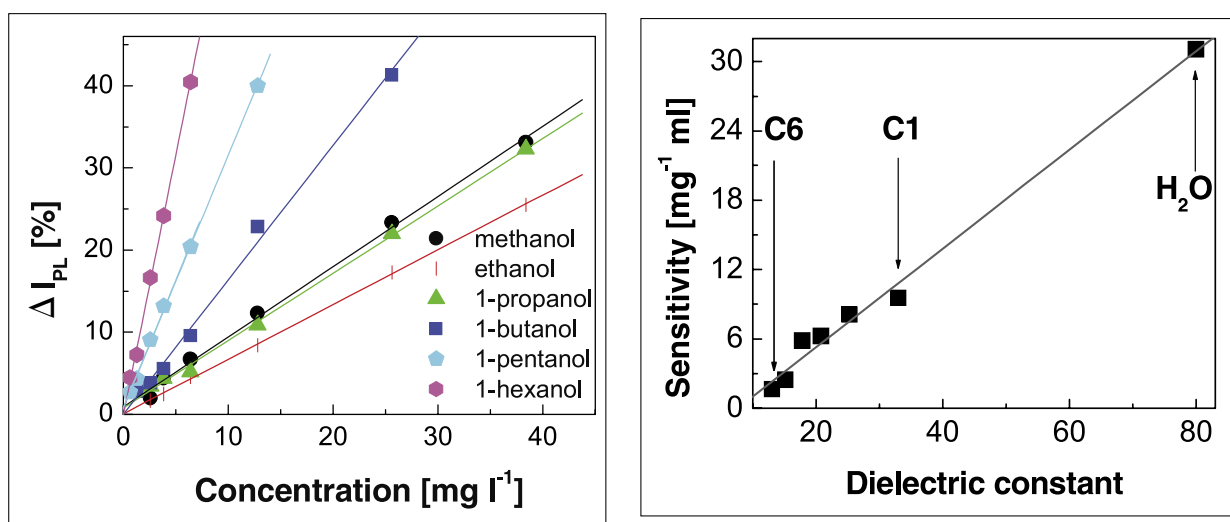

Fig. 5. Sensor response of standard porous silicon as a function of concentration for $n$ alcohols in the gas phase. Emission wavelength $\lambda=700 \mathrm{~nm}$.

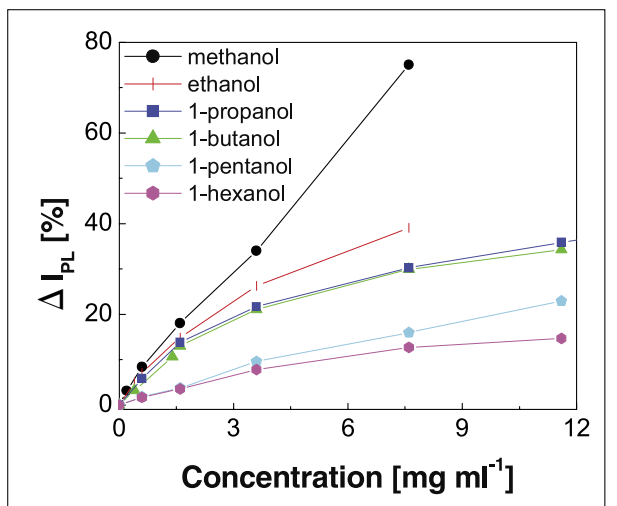

Fig. 6. Sensor response of standard porous silicon as a function of concentration for $\mathrm{n}$-alcohols in the liquid phase. Emission wavelength $\lambda=700 \mathrm{~nm}$.

eters: limit of detection, sensitivity, and linear dynamic range [18].

As it follows from the evaluated quantitative data, the photoluminescence response of PS to analytes in the gas phase is characterized by a nearly monotone increase of sensitivity with the length of alcohol chain; the behavior of sensitivity towards the same analytes in liquid phase is the opposite. Such different sensor behavior can be explained by considering both the factors affecting the extent of luminescence quenching by a given structure and the actual concentration of the analyte in the porous matrix.

Photoluminescence quenching of PS in liquid phase can be explained by the dielectric quenching mechanism [19]. The probability of radiative recombination of excitons in PS depends on the effective dielectric constant inside the porous matrix. With increasing effective dielectric constant the Coulombic force between electron and hole as well as their recombination probability are lowered. Values of sensor sensitivity to studied alcohols in the liquid phase as a function of dielectric constant are presented in Fig. 7. A very good correlation between photoluminescence quenching and dielectric constant of alcohols was observed.
Fig. 7. Dependence of the sensor sensitivity of standard porous silicon as a function of the dielectric constant of linear alcohols in the liquid phase

The behavior of sensor response to linear alcohols in the gas phase is more complex and cannot be explained by the dielectric quenching mechanism only. In PS a broad distribution of pores with diameters of the order of 1-100 $\mathrm{nm}$ is present [20]. Alcohol vapors in the gas phase interact with the porous matrix and their concentration inside the matrix is determined primarily by the capillary condensation effect [21]. The real concentration of alcohol inside the porous matrix is therefore different from the nominal concentration in the gas phase. In a first approximation we can estimate the magnitude of the equilibrium concentration of analyte in the porous matrix by means of a gas phase concentration and analyte vapor pressure. We assume that the analyte concentration in pores for a given analyte gas phase concentration increases with decreasing saturated vapor pressure. The values of sensor response sensitivity as a function of the reciprocal value of saturated vapor pressure are depicted in Fig. 8. The observed correlation explains well why the gas phase sensor sensitivity for 1-hexanol is much higher than for methanol [22].

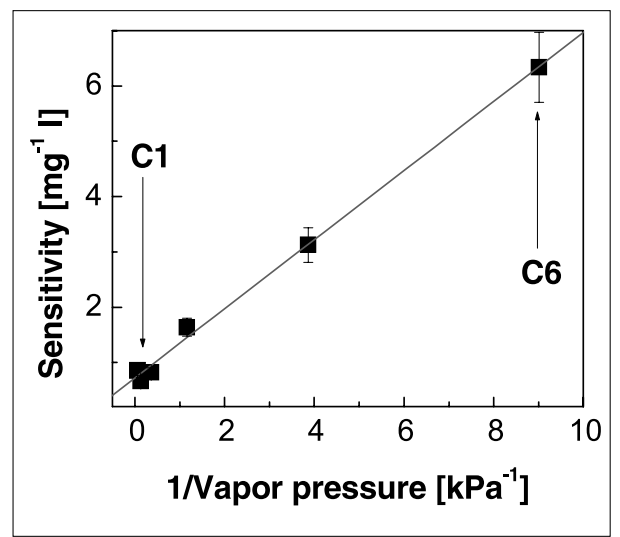

Fig. 8. Dependence of the sensor sensitivity of standard porous silicon on the reciprocal value of vapor pressure of linear alcohols in the gas phase 


\subsection{Sensor Response of Surface Modified Porous Silicon}

Chemical modifications of the PS surface were performed by wet oxidation and hydrosilylation reaction with methyl 10-undecenoate and hemin [18]. We performed measurements of the photoluminescence response with chemically modified samples of PS for the set of $\mathrm{C}_{1}-\mathrm{C}_{6}$ linear alcohols in the gas phase. In comparison to the original PS, the sensor response of the methyl 10-undecenoate-derivatized PS sample was unusually low for lower alcohols. Such observations suggest that methyl 10-undecanoate groups on the PS surface interact with alcohol molecules hindering them from penetration inside the porous matrix. The modified sensor response can be simply quantified by the ratio of sensor sensitivity, $\mathrm{S}_{\mathrm{B}}$, of surface-derivatized PS to sensitivity, $S_{A}$, of as-prepared PS towards the selected analyte. As it follows from the Table, the value of $S_{B} / S_{A}$ monotonically decreases with increasing length of alcohol chain, thus indicating a molecular recognition effect of the surface-modified PS towards n-alcohol vapors. From the structural features of the interacting species we propose that the most probable driving force behind the molecular recognition is the polar interaction between -OH groups of alcohol and $\mathrm{C}=\mathrm{O}$ groups of 10-undecanoate methylester.

Recently we performed further modifications of the porous silicon surface - physical adsorption of cobalt phthalocyanine [23] and electrodeposition of polypyrrole [24]. All these surface modifications significantly altered the photoluminescence sensor response and substantially improved its operational stability.

\section{Conclusion}

The dependence of photoluminescence properties and morphology of porous silicon on fabrication parameters was studied and the influence of current density and electrolyte composition on sample porosity and morphology was determined.
Porous silicon photoluminescence quenching in presence of organic analytes in gas and liquid phases was investigated. The photoluminescence quenching mechanism is consistent with the model of exciton dielectric quenching. This mechanism fully explains the response to various analytes in the liquid phase. The magnitude of the sensor response in the gas phase depends on the thermodynamic equilibrium concentration of the analyte in the porous silicon matrix controlled by the capillary condensation effect.

Derivatization of the PS surface with methyl 10-undecenoate strongly modifies the PL response of the PS sensor in the presence of vapors of lower alcohols as compared to as-prepared PS sensor. The relative change of the PL response scales with the polarity strength of the analyte. Polar interaction between the hydroxyl group of alcohols and the carbonyl group of methyl 10-undecanoate molecules at the PS surface is the dominant mechanism of molecular recognition.

\section{Acknowledgement}

Parts of this work were financially supported by projects GACR203/03/0900, GAUK424/2004/Ch and MSM0021620835.

Received: February 8, 2005

[1] L.T. Canham, Appl. Phys. Lett. 1990, 57, 1046.

[2] F. Koch, V. Petrova-Koch, T. Muschik, $J$. Luminesc. 1993, 57, 271.

[3] L.T. Canham, in 'Properties of Porous Silicon', Ed. L.T. Canham, EMIS Datareview No. 18, INSPEC, London, 1997, p. 44.

[4] M.J. Sailor, in 'Properties of Porous Silicon', Ed. L.T. Canham, EMIS Datareview No. 18, INSPEC, London, 1997, p. 364.

[5] R.L. Smith, S.D. Collins, J. Appl. Phys. 1992, 71, R1.

[6] M. Christophersen, J. Carstensen, S. Rönnebeck, C. Jäger, W. Jäger, H. Föll, J. Electrochem. Soc. 2001, 148, E267.

[7] J.-N. Chazalviel, F. Ozanam, N. Gabouze, S. Fellah, R.B. Wehrspohn, J. Electrochem. Soc. 2002, 149, C511.
[8] M. Christophersen, J. Carstensen, K. Voigt, H. Föll, Phys. Stat. Sol. (a) $\mathbf{2 0 0 3}$ 197, 34.

[9] S. Lust, C. Levy-Clement, J. Electrochem. Soc. 2002, 149, C338.

[10] D.J. Blackwood, Y. Zhang, Electrochimica Acta 2003, 48, 623.

[11] T. Holec, T. Chvojka, I. Jelínek, J. Jindřich, I. Němec, I. Pelant, J. Valenta, J. Dian, Mat. Sci. Eng. C 2002, 19, 251.

[12] M.J. Sailor, E.J. Lee, Adv. Mater. 1997, 9 , 783.

[13] J.M. Buriak, Chem. Rev. 2002, 102, 1271.

[14] W. Göpel, in 'Sensors', Vol. 8, Eds. H. Meixner, R. Jones, WCH Weinheim, 1995, p. 295.

[15] D. Brumhead, L.T. Canham, D.M. Seekings, P.J. Tufton, Electrochim. Acta $\mathbf{1 9 9 3}$ 38, 191.

[16] J. Dian, A. Macek, D. Nižňanský, I. Němec, V. Vrkoslav, T. Chvojka, I. Jelínek, Appl. Surf. Sci. 2004, 238, 169.

[17] B. Bessaïs, O. Ben Younes, H. Ezzaouia, N. Mliki, M.F. Boujmil, M. Oueslati, R. Bennaceur, J. Luminesc. 2000, 90, 101.

[18] T. Chvojka, T. Holec, I. Jelínek, I. Němec, J. Jindřich, M. Lorenc, J. Koutníková, V. Král, J. Dian, in 'Photonics, Devices and Systems II', Eds. M. Hrabovský, D. Senderáková, P. Tománek, Proc. SPIE Vol. 5036, 2003, 51.

[19] S. Fellah. R.B. Wehrspohn, N. Gabouze, F. Ozanam, J.N. Chazalviel, J. Luminesc. 1999, 80, 109.

[20] L.T. Canham, in 'Properties of Porous Silicon', Ed. L.T. Canham, EMIS Datareviews Series No. 18, INSPEC, London 1997, p. 83.

[21] S.J. Gregg, K.S.W. Singh, 'Adsorption, surface area and porosity', Academic Press, London 1967.

[22] T. Chvojka, V. Vrkoslav, I. Jelínek, J. Jindřich, M. Lorenc, J. Dian, Sens. \& Actuators B 2004, 100, 246.

[23] V. Vrkoslav, I. Jelínek, M. Matocha, V. Král, J. Dian, submitted to Mat. Sci. Eng. C.

[24] V. Vrkoslav, I. Jelínek, G. Broncová, H. Březnová, V. Král, J. Dian, Chem. Lett. 2004, 98, 1039.

\begin{tabular}{llll} 
Analyte & $\begin{array}{l}\mathrm{S}_{\mathrm{A}} \\
{\left[\mathrm{g}^{-1} \mathrm{I}\right]}\end{array}$ & $\begin{array}{l}\mathrm{S}_{\mathrm{B}} \\
{\left[\mathrm{g}^{-1} \mathrm{l}\right]}\end{array}$ & $\mathrm{S}_{\mathrm{B}} / \mathrm{S}_{\mathrm{A}}$ \\
\hline methanol & 0.577 & 0.195 & 0.251 \\
ethanol & 0.545 & 0.174 & 0.216 \\
1-propanol & 0.921 & 0.271 & 0.357 \\
1-butanol & 0.949 & 0.427 & 0.481 \\
1-pentanol & 1.128 & 0.541 & 0.462 \\
1-hexanol & 1.317 & 0.600 & 0.533 \\
\hline
\end{tabular}

